A formal joint count remains the most specific measure of RA disease activity; however, RAPID3, which takes fewer than 10s for the physician to calculate and provides information supplementary to that obtained from a qualitative examination, might prove valuable for tracking patient status in a typical clinical setting.

Original article Pincus T et al. (2008) RAPID3 (Routine Assessment of Patient Index Data 3), a rheumatoid arthritis index without formal joint counts for routine care: proposed severity categories compared to disease activity score and clinical disease activity index categories. J Rheumatol 35: 2136-2147

\section{Pegloticase reduces serum urate levels in patients with treatment-refractory gout}

Gout patients who do not attain target serum urate levels of $\leq 6 \mathrm{mg} / \mathrm{dl}$ with conventional uratelowering therapies are defined as having treatment-refractory gout. A proposed new therapy, urate oxidase (pegloticase), converts poorly soluble urate to allantoin, which is highly soluble and easily excreted. Following successful single-dose trials, Sundy and colleagues' phase II trial has demonstrated that multiple pegloticase infusions are effective in reducing and maintaining plasma urate at or below target levels in patients with treatmentrefractory gout.

This open-label, parallel-group study included 41 patients from 9 US centers who had hyperuricemia and treatment-refractory gout or contraindications to conventional therapy (mean disease duration 14.4 years). Participants were randomly allocated to one of four 12-14-week pegloticase infusion regimens, which all showed statistically similar efficacy in achieving target plasma urate levels. This treatment did not prevent flare. Infusion-associated adverse events motivated 12 of 15 withdrawals from the study, so the protocol was amended from pegloticase administration in $100 \mathrm{ml}$ phosphate-buffered saline over $30 \mathrm{~min}$ to $200 \mathrm{ml}$ over $1 \mathrm{~h}$.

The study demonstrated that pegloticase might be immunogenic at doses low enough to avoid accumulation, which suggests that its efficacy would decrease rapidly. Longterm, placebo-controlled trials including larger numbers of patients are required to determine the optimal pegloticase regimen, durability of response to pegloticase, adverse event rate and this treatment's potential to reduce clinical manifestations of gout.

Original article Sundy JS et al. (2008) Reduction of plasma urate levels following treatment with multiple doses of pegloticase (polyethylene glycol-conjugated uricase) in patients with treatment-failure gout: results of a phase II randomized study. Arthritis Rheum 58: 2882-2891

\section{Belimumab is well tolerated and depletes B cells effectively in patients with SLE}

B cells have important pathogenic roles in systemic lupus erythematosus (SLE): they produce autoantibodies, secrete inflammatory cytokines and might promote expansion of autoreactive $\mathrm{T}$ cells via antigen presentation. Several treatments that target B cells have, therefore, been investigated in patients with SLE. Furie et al. now report favorable results for their phase I trial of belimumab - a fully human, neutralizing, monoclonal antibody against B-lymphocyte stimulator - which both stimulates proliferation and inhibits apoptosis of B cells.

This multicenter, randomized, double-blind, dose-escalation study of belimumab involved 70 patients with mild-to-moderate SLE, of whom 36 received a single infusion of belimumab (at 1, 4, 10 or $20 \mathrm{mg} / \mathrm{kg}$ ) or placebo, while the other 34 received two such infusions, 21 days apart. Patients were monitored for 84 days after their last infusion. Belimumab had linear pharmacokinetics across this dose range, and a half-life of approximately 14 days, which suggested that dosing intervals of 28 days were appropriate for future studies. The incidence of adverse events and laboratory abnormalities was similar in treatment and placebo groups. Compared with placebo, belimumab significantly reduced patients' median percentages of CD20+ $\mathrm{B}$ cells by up to $47 \%$.

Phase II trials to assess the safety and clinical activity of belimumab in patients with SLE and rheumatoid arthritis are under way.

\footnotetext{
Original article Furie R et al. (2008) Biologic activity and safety of belimumab, a neutralizing anti-B-lymphocyte stimulator (BLyS) monoclonal antibody: a phase 1 trial in patients with systemic lupus erythematosus. Arthritis Res Ther 11: R109
} 